\title{
Endosialin expression in side populations in human sarcoma cell lines
}

\author{
CECILE ROULEAU, JOSE SANCHO, JUANITA CAMPOS-RIVERA and BEVERLY A. TEICHER
}

Genzyme Corporation, Framingham, MA 01701, USA

Received March 4, 2011; Accepted July 15, 2011

DOI: $10.3892 / 01.2011 .478$

\begin{abstract}
The Hoechst 33342 exclusion side population (SP) assay is a validated method used to identify cells with stem cell-like properties. When isolated from tumors, SP cells have been shown to have high malignant potential. SPs have been found in both carcinomas and sarcomas. The molecular profile of sarcoma SP is poorly understood. The purpose of the present study was to determine whether endosialin is a suitable therapeutic target for sarcomas. Six cell lines (HT-1080 fibrosarcoma, SJSA-1 and HOS osteosarcoma, A-673 and SK-ES-1 Ewing sarcoma) were used for the SP analysis. Flow cytometry was used to count and examine the cells. Results showed for the first time that endosialin (CD248), which was previously identified as a sarcoma marker, is expressed in sarcoma SP cells. This observation supports the hypothesis that endosialin is a promising therapeutic target for sarcomas.
\end{abstract}

\section{Introduction}

Central to our understanding of cancer progression is the recognition of tumor heterogeneity and the finding that cells within a tumor vary in their malignant potential $(1,2)$. The search for the highly malignant cell, i.e., the cell that initiates primary tumor growth, escapes therapy and repopulates the tumor and/or the motile and invasive cell that survives in circulation and colonizes distant organs, has led to the ongoing characterization of the metastatic cell, tumor-initiating cell or cancer stem cell, as well as the circulating tumor cell, all of which exhibit highly malignant and partially overlapping phenotypes (3-6).

The Hoechst 33342 exclusion side population (SP) assay is a method used to identify and isolate cells with highly malignant properties. SPs were first identified by Goodell et al (7) in the process of isolating cycling vs. quiescent bone marrow cells by flow cytometry using the DNA binding dye Hoechst 33342 . The investigators identified a small distinct cell subpopulation

Correspondence to: Dr Beverly A. Teicher, Developmental Therapeutics Program, National Cancer Institute, 6130 Executive Boulevard, Rock ville, MD 20852, USA

E-mail: beverly.teicher@nih.gov

Key words: side populations, cancer stem cells, sarcoma marker with high dye efflux capacity, enriched in hematopoietic stem cell markers and in vivo bone marrow reconstitution activity. This population was termed 'side population' (SP) as it appeared on dual-wavelength cytograms capturing emission in the red and blue ranges of the spectrum. Disappearance of the SP profile upon exposure to verapamil indicated that the effect was mediated by multidrug resistance proteins (7). Expression of high cell surface MDR1/P-glycoprotein and/or BCRP1/ ABCG2 was subsequently found to be a key feature of the SP phenotype $(8,9)$. Since the initial identification in bone marrow, SP cells have been identified across tissue types, including muscle, breast, lung, liver, brain, skin, heart and kidney, where they have regenerative and stem cell-like properties $(10,11)$.

The Hoechst 33342 exclusion SP phenotype is relevant to cancer since drug efflux in malignant cells is a mechanism of chemoresistance that provides survival advantage and promotes tumor recurrence and disease progression (12). Similarly, the normal stem cell phenotype, characterized by a lack of differentiation and by the capacity for self-renewal and repopulation, bears some similarity to the malignant cell phenotype and malignant progression (13). SP cells have been detected in various types of cancer, including leukemia, glioma, medulloblastoma, hepatocellular, breast, prostate, thyroid, colorectal and ovarian carcinoma. In general, SP cells have a superior tumorigenic potential compared to non-SP cells, as determined by their ability to initiate tumors in immunodeficient mice $(10,11)$.

The first observation of SP cells in tumors of mesenchymal origin was reported by Wu et al (14) in 2007. SP cells were detected in 26/29 human sarcomas and there was a positive correlation between the percentage of SP cells in the tumor and the grade of the tumor. Wu et al also found that SP cells had greater tumor-initiating ability upon implantation in NOD/SCID mice, with lower numbers of SP cells required for tumor initiation compared to non-SP cells. Furthermore, SP cells had a more efficient tumor uptake and a larger tumor as compared to non-SP cells. Notably, only SP cells were capable of generating tumors containing both SP and non-SP cells, demonstrating the unique ability of SP cells to recapitulate the phenotype of the original tumor. In addition, only SP cells retained tumor-initiating ability upon passaging from animal to animal, demonstrating the unique ability of SP cells to self-renew (14). Subsequently, Komuro et al identified SP cells in human pediatric cancer cell lines, including sarcoma cell lines (15). In 2009, Murase et al confirmed the greater tumorigenic potential of SP cells in vivo and proved 
the greater clonogenic potential of sarcoma SP cells in vitro in the spheroid colony formation assay (16).

The molecular characterization of tumor SP cells has begun to yield novel therapeutic approaches and pre-clinical advances, but the gene expression profile of sarcoma SP cells remains to be elucidated $(10,11)$. Few reports detailing molecular characterization of sarcoma SP cells are currently available. Using a bone malignant fibrous histiocytoma cell line, Murase et al identified 23 transcripts that were up-regulated in SP cells compared to the bulk tumor cell population. Among the transcripts found at higher levels in the SP cells was the efflux pump ABCG2, which was also up-regulated in the SP cell fraction of the SK-ES-1 Ewing sarcoma cell line $(16,17)$.

Endosialin is a novel cell surface protein detected in mesenchymal tumors, as well as perivascular, stromal and malignant cells (18-20). We previously conducted a survey of endosialin protein expression by immunohistochemistry in clinical specimens of sarcoma (20). Endosialin expression was frequent in clinical sarcoma specimens and reached high levels: 70 of 86 (81\%) sarcomas were positive for endosialin, with $44(51 \%)$ exhibiting at least $50 \%$ coverage of the three immunoreactive cell types as a whole. Staining intensity was scored on the scale $0,1+, 2+$ and $3+$; all nine sarcoma subtypes surveyed included specimens reaching a staining intensity of $2+$ and $3+$. Endosialin is also expressed in progenitor cells, such as endothelial precursor and mesenchymal stem cells (21-24).

\section{Materials and methods}

Materials. Hoechst 33342, verapamil and propridium iodide (PI) were purchased from Sigma-Aldrich (St. Louis, MO, USA).

Cells. All cells were of human origin. The HT-1080 fibrosarcoma, SJSA-1 and HOS osteosarcoma, A-673 and SK-ES-1 Ewing sarcoma cell lines were obtained from the American Type Culture Collection (ATCC, Manassas, VA, USA). The MG-63 osteosarcoma cell line was obtained from the Riken Bioresource Center (Ibaraki, Japan). The cells were propagated in RPMI supplemented with $10 \%$ heat-inactivated fetal bovine serum (FBS) (Invitrogen, Carlsbad, CA, USA).

Antibody. The fully human anti-endosialin antibody used for flow cytometry was generated through a partnership with Kyowa Hakko Kirin Co., Ltd., Takasaki, Japan, as previously described (20).

Side population analysis by flow cytometry. The cells were detached in warm Versene (PBS-EDTA; Invitrogen). Warm RPMI, 2\% FBS, $10 \mathrm{mM}$ HEPES was added once the cells were detached. The cells were centrifuged for $5 \mathrm{~min}$ at room temperature and suspended in warm HBSS, 2\% FBS, $10 \mathrm{mM}$ HEPES at a concentration of 5 million cells $/ 200 \mu \mathrm{l}$. Five million cells $/ 200 \mu \mathrm{l}$ were used per tube for staining. Hoechst $33342(10 \mu \mathrm{g} / \mathrm{ml})$ was added to each tube in the presence or absence of verapamil $(100$ or $500 \mu \mathrm{M})$. The cells were incubated in the presence of Hoechst 33342 and in the presence or absence of verapamil for $2 \mathrm{~h}$ at $37^{\circ} \mathrm{C}$ with agitation in the dark, after which the tubes were transferred to ice. Anti-endosialin antibody $(20 \mu \mathrm{g} / \mathrm{ml})$ was added and incubated for $45 \mathrm{~min}$ on ice in cold HBSS, 2\% FBS, $10 \mathrm{mM}$
HEPES. The cells were washed once in cold HBSS, 2\% FBS, $10 \mathrm{mM}$ HEPES and suspended in $200 \mu \mathrm{l}$ cold HBSS, 2\% FBS, 10 mM HEPES. Phycoerythrin-labeled anti-human IgG $\mathrm{F}\left(\mathrm{ab}^{\prime}\right)_{2}$, (Jackson ImmunoResearch Laboratories, Inc., West Grove, PA, USA) secondary antibody was added at a dilution of 1/40 and incubated on ice for $45 \mathrm{~min}$. The cells were washed once in cold HBSS, 2\% FBS, 10 mM HEPES and suspended in $500 \mu \mathrm{l}$ cold HBSS, $2 \%$ FBS, $10 \mathrm{mM}$ HEPES. PI $(0.5 \mu \mathrm{g} / \mathrm{ml})$ was added immediately prior to the acquisition. Cells were analyzed by flow cytometry with a 4-laser equipped SORP LSRII (Becton-Dickinson Biosciences, San Jose, CA, USA). PI was excited by 488-nm laser and the resulting fluorescence was detected using a filter 610/20 with a LP dichroid mirror 610LP. Hoechst 33342 was excited with a $355 \mathrm{~nm}$ UV laser, and its fluorescence was measured dually: Hoechst Red with 605/40 filter and Hoechst Blue with 450/40. PE was excited by a $488-\mathrm{nm}$ laser and the resulting fluorescence was detected using a 575/26 filter with a 550 LP dichroid. Data analysis was conducted in Flow Jo (Tree Star Inc., Ashland, OR, USA).

\section{Results}

We previously studied endosialin expression by flow cytometry in 37 human sarcoma cell lines and detected the expression thereof in 18 of the 37 cell lines (25). In the present study, six of the 37 human sarcoma cell lines, the HT-1080 fibrosarcoma cell line, the A-673 and SK-ES-1 Ewing sarcoma cell lines and the SJSA-1, MG-63 and HOS osteosarcoma cell lines, were examined to determine whether or not SP cells were detected in the lines, and to determine whether the SP cells expressed endosialin.

SP cells were identified upon simultaneous exposure to Hoechst 33342 and verapamil for $2 \mathrm{~h}$. The combination of Hoechst 33342 and verapamil was toxic to the cells as measured by the PI uptake. The decrease in the number of viable PI-negative cells was cell-line-dependent and verapamil concentration-dependent: a modest decrease in viability was observed with $100 \mu \mathrm{M}$ verapamil, whereas a more profound decrease in viability was observed with $500 \mu \mathrm{M}$ verapamil. The cytotoxicity observed resulted in the lowest viability in HOS cells (33\% viability) and in SK-ES-1 cells (35\% viability), and the highest viability in HT-1080 cells (65\% viability) at the higher verapamil concentration (Fig. 1). These results are consistent with published observations showing that verapamil is cytotoxic towards cells in culture $(26,27)$.

Decreased viability was also observed in the absence of verapamil and in the presence of Hoechst 33342 alone. The cytotoxicity of Hoechst 33342 varied among the cell lines with the lowest viability observed in the SK-ES-1 cells (46\% viability) and HOS cells (55\% viability), and the highest viability observed in SJSA-1 cells (81\% viability) and HT-1080 cells (78\% viability) (Fig. 1). The results are consistent with published observations showing that Hoechst 33342 is cytotoxic towards cells in culture $(28,29)$.

SP cells were identified among viable PI-negative cells as verapamil-sensitive cells with low Hoechst 33342 content. Sensitivity to the efflux-blocking activity of verapamil varied between cells. Although verapamil $(500 \mu \mathrm{M})$ induced a reduction in the number of cells with low Hoechst 33342 content in all six cell lines tested, only two of the six, the MG-63 and 


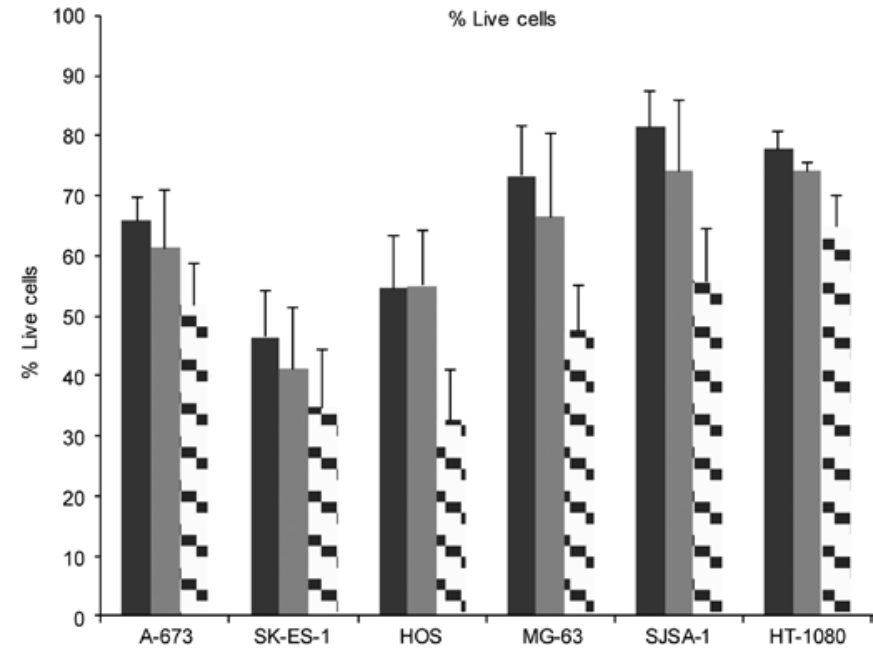

Figure 1. Effect of Hoechst 33342 and verapamil on cell viability. Six human sarcoma cell lines were exposed to Hoechst 33342 for $2 \mathrm{~h}$ in the absence of verapamil (solid black bar), in the presence of $100 \mu \mathrm{M}$ verapamil (solid grey bar) or of $500 \mu \mathrm{M}$ verapamil (checked bar). The percentage of live cells was determined by flow cytometry by subtracting the sub-G0 DNA-content cells. Combination of three independent experiments is shown. Error bars: standard error of the mean.

SJSA-1 osteosarcoma cell lines, were sensitive to $100 \mu \mathrm{M}$ verapamil (Fig. 2A and B). The SJSA-1 osteosarcoma cell line was particularly sensitive to verapamil, with a similar percentage of SP cells detected in the presence of 100 and $500 \mu \mathrm{M}$ verapamil ( 0.12 and $0.09 \%$, respectively). The concentrations of verapamil used in this study are similar to those used by Komuro et al (2007) who reported verapamil sensitivity between 50 and 500 $\mu \mathrm{M}$ in a number of human sarcoma cell lines (15).

As a result of the lack of sensitivity of 4 of the 6 cell lines to $100 \mu \mathrm{M}$ verapamil, the $500 \mu \mathrm{M}$ concentration was selected as the concentration for identification and gating of verapamilsensitive cells. The highest percentage of SP cells was observed in SK-ES-1 Ewing sarcoma cells (0.73\%), followed by MG-63 osteosarcoma (0.60\%), HOS osteosarcoma (0.39\%), SJSA-1 osteosarcoma (0.37\%), HT-1080 fibrosarcoma (0.24\%) and A-673 Ewing sarcoma cells (0.17\%) (Fig. 2A and B). The results obtained for the SK-ES-1 cell line are similar to previously reported results, in which SP cells were found to comprise $1.2 \%$ of SK-ES-1 cell populations (17). Our results are consistent with those reported by Komuro et al in Ewing sarcoma cell lines (15).

We determined whether SP cells maintained endosialin positivity in the four cell lines for which the main population was previously determined to be endosialin-positive: A-673 and SK-ES-1 Ewing sarcoma cells, and HOS and SJSA-1 osteosarcoma cells. Results indicated that the percentage of endosialin-positive cells was similar in SP and non-SP cells: in A-673, $98 \%$ of SP cells and $99 \%$ of non-SP cells were endosialin-positive; in SK-ES-1, 96\% of SP cells and $89 \%$ of non-SP cells were endosialin-positive; in HOS, $98 \%$ of SP cells and $94 \%$ of non-SP cells were endosialin-positive; and in SJSA-1 $98 \%$ of SP cells and $96 \%$ of non-SP cells were endosialin-positive (Fig. 3A). Not only was the percentage of endosialin-positive cells similar between SP cells and non-SP cells, but the level of endosialin expression was also similar between SP cells and non-SP cells as evidenced by the median fluorescence intensity (Fig. 3B).

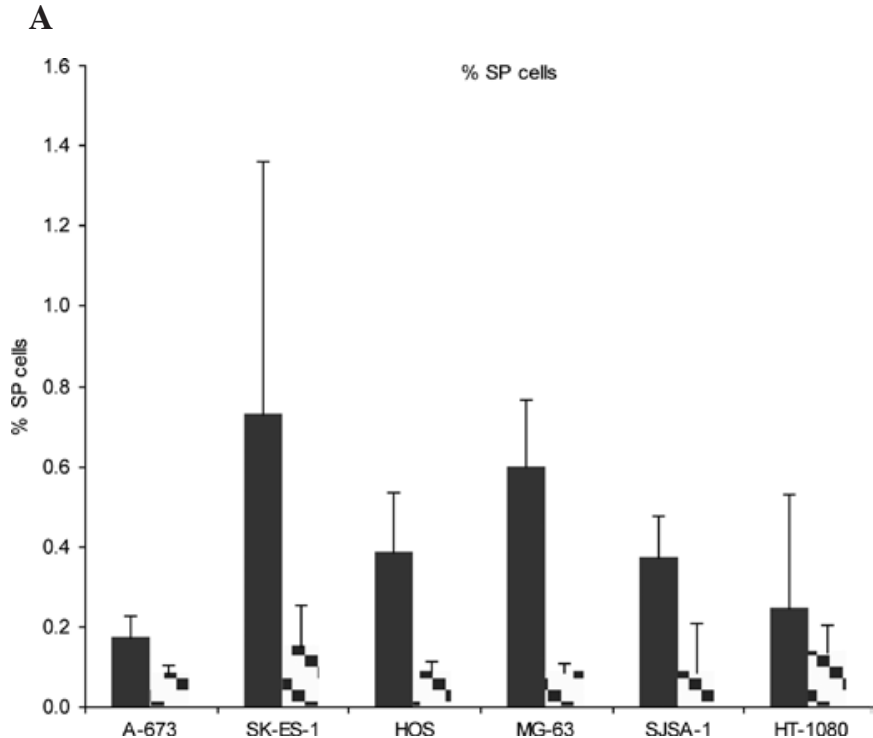

B

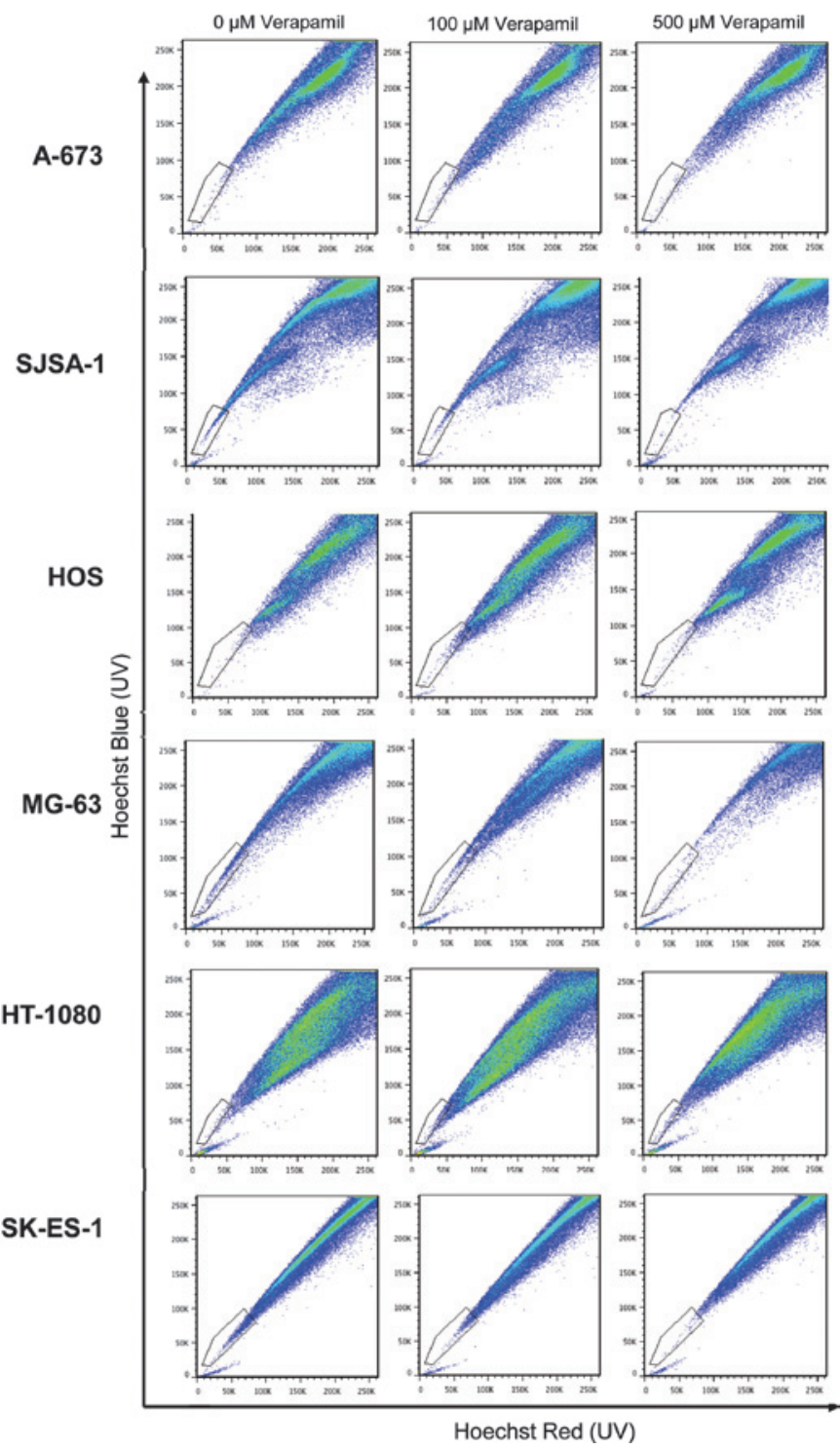

Figure 2. Percentage of SP cells. (A) Cells were exposed to Hoechst 33342 for $2 \mathrm{~h}$ in the absence of verapamil (solid black bar) or in the presence of $500 \mu \mathrm{M}$ verapamil (checked bar). SP was determined through red and blue Hoechst fluorescence. (B) Representative staining. Combination of three independent experiments is depicted. Error bars: standard error of the mean. 
A

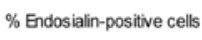

"(SP) A-673 $\boldsymbol{m}$ (Non SP) A-673

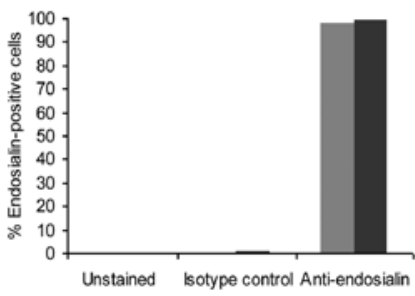

\% Endosialin-positive cells

= (SP) HOS $\mathbf{m}$ (Non SP) HOS

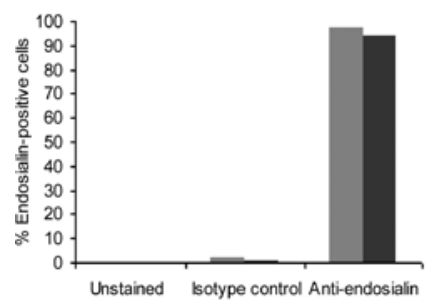

$\%$ Endosialn-positive cells

= (SP) SJSA-1 $\mathbf{m}$ (Non SP) SJSA-1

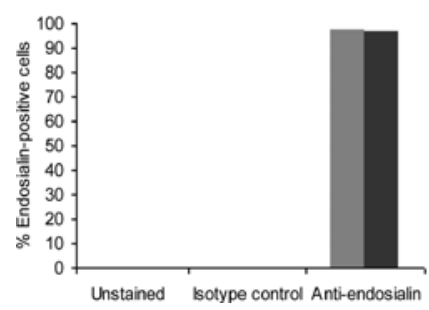

\% Endosialin-positive cells

= (SP) SK-ES-1 $\mathbf{m}$ (Non SP) SK-ES-1
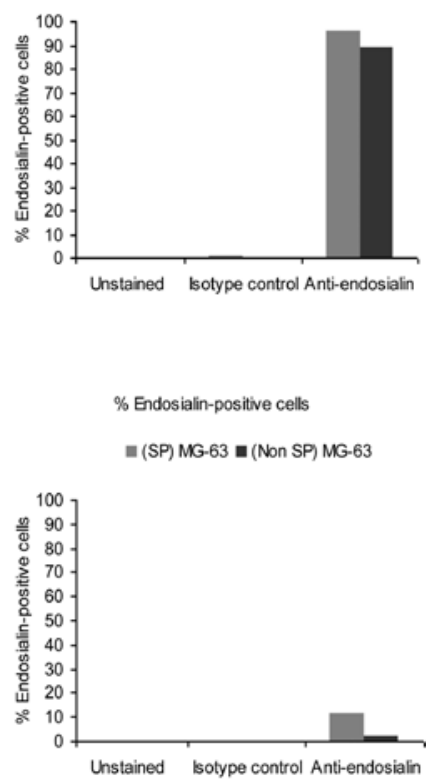

= (SP) HT-1080 $\mathbf{m}$ (Non SP) HT-1080

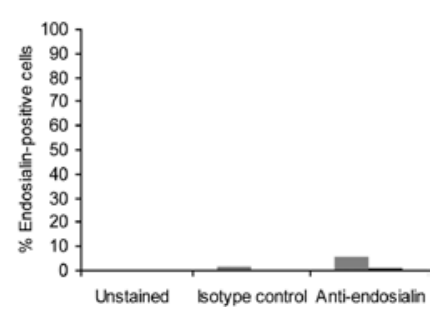

A-673

MG-63

HOS

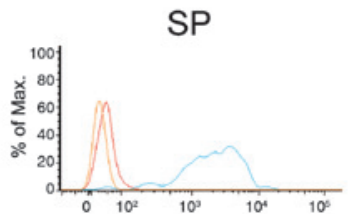

SJSA-1
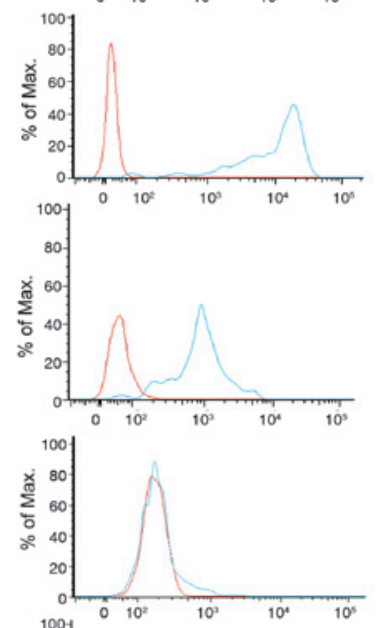

HT-1080

B

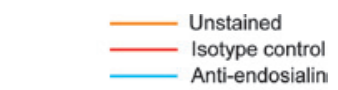

Non-SP
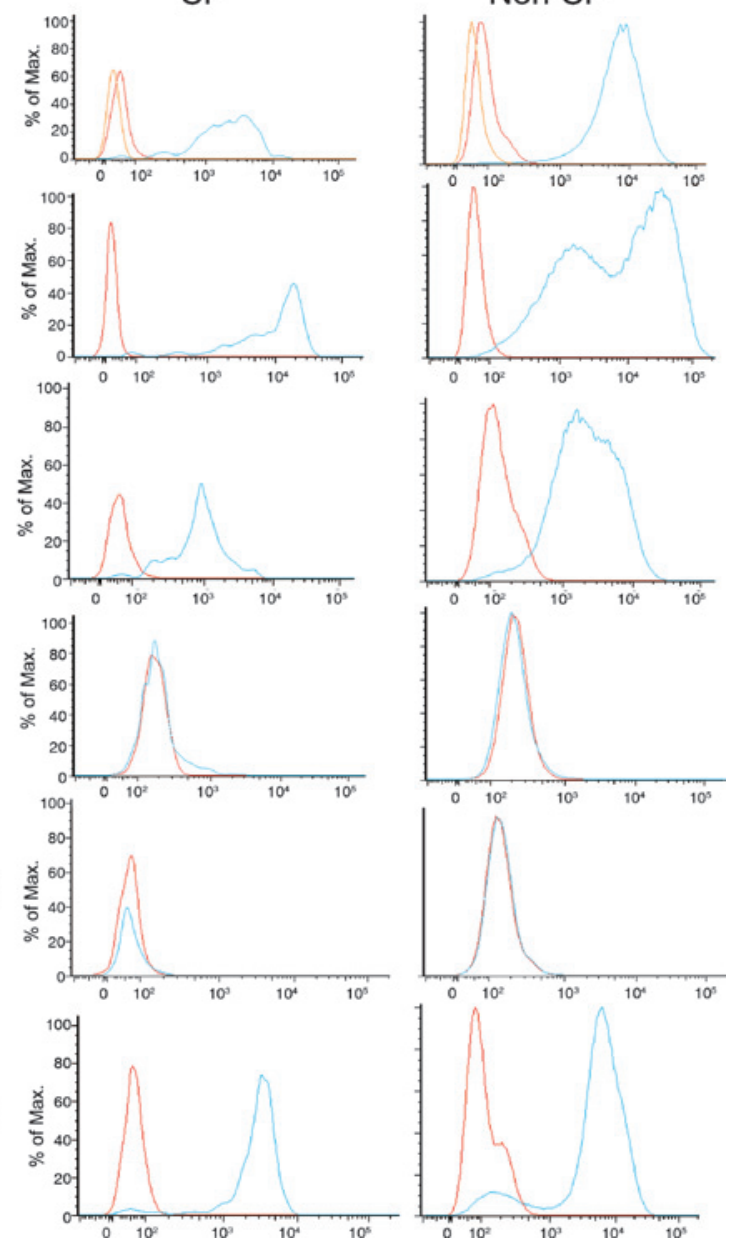

Figure 3. Endosialin expression in SP cells and non-SP cells. (A) Percentage of endosialin-positive cells in SP cells and non-SP cells. (B) Representative histograms showing endosialin staining fluorescence intensity in SP cells and non-SP cells.

For the two cell lines for which the main population had previously been determined to be negative for endosialin, the MG-63 osteosarcoma and HT-1080 fibrosarcoma cell lines, SP cells and non-SP cells were found to be similarly negative, with similar median fluorescence intensities (Fig. 3B).

\section{Discussion}

SK-ES-1, A-673, SJSA-1 and HT-1080 cells are tumorigenic in immunodeficient mice, whereas the HOS and MG-63 cell lines are not (20,25,30-35). In 2008, Wu and Alman stated that a correlation between the percentage of SP cells and tumorigenicity had never been demonstrated (11). The detection of SP cells in two non-tumorigenic cell lines, HOS and MG-63, and the detection of lower percentages of SP cells in highly tumorigenic cell lines, such as A-673, compared to non-tumorigenic cell lines, also fail to show any correlation between the percentage of SP cells and in vivo growth in cell lines. Studies of correlation between the percentage of SP cells and tumorigenicity of human cell lines are intrinsically limited by the xenograft nature of the in vivo experiment, where the mouse microenvironment may selectively promote the growth of some clones but not others $(11,36)$. It is possible that the tumorigenicity of the original tumors from which the HOS and MG-63 cell lines were derived is counteracted by unknown factors in the murine host $(37,38)$.

However, a correlation between the percentage of SP cells and tumor grade was compellingly established by $\mathrm{Wu}$ et al in 2007 for mesenchymal tumors freshly resected from human patients (14). SP cells were separated from non-SP cells and both SP and non-SP cells formed tumors in mice. However, only SP cells remained tumorigenic through serial transplantation in mice, a fact attributed to the unique ability of SP cells to self-renew. These data demonstrated that SP and non-SP cells have different growth properties in vivo. Whether or not HOS SP cells or MG-63 SP cells have tumorigenic potential, while the parent cell lines do not, is a noteworthy possibility that should be tested experimentally. If HOS SP and MG-63 $\mathrm{SP}$ cells are tumorigenic in vivo, then the mechanisms by which non-SP cells repress SP cell tumorigenicity when co-implanted in mice, as is the case when the parent line is implanted, would need to be elucidated.

Identifying markers of highly malignant cells is crucial for the development of new effective therapeutics. Since SP cells have been shown to be enriched in malignant potential, SP cell molecular characterization may allow the identification 
of useful diagnostic markers and/or therapeutic targets. Not all sarcoma clinical specimens or sarcoma cell lines express endosialin $(20,25)$. The current data indicate that, in sarcoma cell lines that are positive for endosialin, SP cells maintain endosialin positivity.

In conclusion, we have reported for the first time that endosialin is expressed in sarcoma SP cells, thereby reinforcing the hypothesis that endosialin is a suitable therapeutic target for sarcomas. However, additional endosialin-positive cell lines should be screened to determine whether retention of endosialin positivity in sarcoma SP cells is a frequent event, and SP cells should be isolated from clinical specimens and assessed for endosialin expression.

\section{Acknowledgements}

The authors conducted the study as employees of Genzyme Corporation. Cecile Rouleau: Conception and design, collection and assembly of data, data analysis and interpretation, manuscript writing. Jose Sancho: Conception and design, collection and assembly of data, data analysis and interpretation. Juanita Campos-Rivera: Conception and design, collection and assembly of data, data analysis and interpretation. Beverly A. Teicher: Conception and design, collection and assembly of data, data analysis and interpretation, manuscript writing, final approval of manuscript and project supervision.

\section{References}

1. Marusyk A and Polyak K: Tumor heterogeneity: causes and consequences. Biochim Biophys Acta 1805: 105-117, 2010.

2. Fidler IJ: Tumor heterogeneity and the biology of cancer invasion and metastasis. Cancer Res 38: 2651-2660, 1978.

3. Nguyen DX, Bos PD and Massagué J: Metastasis: from dissemination to organ-specific colonization. Nat Rev Cancer 9: 274-284, 2009.

4. Bansal N and Banerjee D: Tumor initiating cells. Curr Pharm Biotechnol 10: 192-196, 2009.

5. Mueller MT, Hermann PC and Heeschen C: Cancer stem cells as new therapeutic target to prevent tumour progression and metastasis. Front Biosci 2: 602-613, 2010.

6. Maheswaran S and Haber DA: Circulating tumor cells: a window into cancer biology and metastasis. Curr Opin Genet Dev 20: 96-99, 2010.

7. Goodell MA, Brose K, Paradis G, et al: Isolation and functional properties of murine hematopoietic stem cells that are replicating in vivo. J Exp Med 183: 1797-1806, 1996.

8. Bunting KD, Zhou S, Lu T, et al: Enforced P-glycoprotein pump function in murine bone marrow cells results in expansion of side population stem cells in vitro and repopulating cells in vivo. Blood 96: 902-909, 2000.

9. Zhou S, Schuetz JD, Bunting KD, et al: The ABC transporter Bcrp1/ABCG2 is expressed in a wide variety of stem cells and is a molecular determinant of the side-population phenotype. Nat Med 7: 1028-1034, 2001.

10. Moserle L, Ghisi M, Amadori A, et al: Side population and cancer stem cells: therapeutic implications. Cancer Lett 288: 1-9, 2010.

11. Wu C and Alman BA: Side population cells in human cancers Cancer Lett 268: 1-9, 2008

12. Liu FS: Mechanisms of chemotherapeutic drug resistance in cancer therapy - a quick review. Taiwan J Obstet Gynecol 48: 239-244, 2009.

13. Reya T, Morrison SJ, Clarke MF, et al: Stem cells, cancer, and cancer stem cells. Nature 414: 105-111, 2001.

14. Wu C, Wei Q, Utomo V, et al: Side population cells isolated from mesenchymal neoplasms have tumor initiating potential. Cancer Res 67: 8216-8222, 2007.

15. Komuro H, Saihara R, Shinya M, et al: Identification of side population cells (stem-like cell population) in pediatric solid tumor cell lines. J Pediatr Surg 42: 2040-2045, 2007.
16. Murase M, Kano M, Tsukahara T, et al: Side population cells have the characteristics of cancer stem-like cells/cancer-initiating cells in bone sarcomas. Br J Cancer 101: 1425-1432, 2009.

17. Yang M, Zhang R, Yan M, et al: Detection and characterization of side population in Ewing's sarcoma SK-ES-1 cells in vitro. Biochem Biophys Res Commun 391: 1062-1066, 2010.

18. Rettig WJ, Garin-Chesa P, Healey JH, et al: Identification of endosialin, a cell surface glycoprotein of vascular endothelial cells in human cancer. Proc Natl Acad Sci USA 89: 10832-10836, 1992.

19. Dolznig H, Schweifer N, Puri C, et al: Characterization of cancer stroma markers: in silico analysis of an mRNA expression database for fibroblast activation protein and endosialin. Cancer Immun 5: 10, 2005.

20. Rouleau C, Curiel M, Weber W, et al: Endosialin protein expression and therapeutic target potential in human solid tumors: sarcoma versus carcinoma. Clin Cancer Res 14: 7223-7236, 2008.

21. Bagley RG, Honma N, Weber W, et al: Endosialin/TEM 1/ CD248 is a pericyte marker of embryonic and tumor neovascularization. Microvasc Res 76: 180-188, 2008.

22. Bagley RG, Rouleau C, St Martin T, et al: Human endothelial precursor cells express tumor endothelial marker 1/endosialin/ CD248. Mol Cancer Ther 7: 2536-2546, 2008.

23. Bagley RG, Weber W, Rouleau C, et al: Human mesenchymal stem cells from bone marrow express tumor endothelial and stromal markers. Int J Oncol 34: 619-627, 2009.

24. Bagley RG: Endosialin: from vascular target to biomarker for human sarcomas. Biomark Med 3: 589-604, 2009.

25. Rouleau C, Smale R, Jones C, et al: Characterizing models for personalized medicine: endosialin in sarcoma from cultured cells to mice to humans. Proceedings of the American Association for Cancer Research/National Cancer Institute/ European Organization for Research and Treatment of Cancer Nov 15-19; Boston, USA, 2009.

26. Cao QZ, Niu G and Tan HR: In vitro growth inhibition of human colonic tumor cells by Verapamil. World J Gastroenterol 11: 2255-2259, 2005.

27. Schmidt WF, Huber KR, Ettinger RS, et al: Antiproliferative effect of verapamil alone on brain tumor cells in vitro. Cancer Res 48: 3617-3621, 1988.

28. Siemann DW and Keng PC: Cell cycle specific toxicity of the Hoechst 33342 stain in untreated or irradiated murine tumor cells. Cancer Res 46: 3556-3559, 1986.

29. Machaliński B, Wiszniewska B, Baśkiewicz M, et al: In vivo and in vitro studies on the toxicity of Hoechst 33342 (Ho342). Implications for employing Ho342 for the isolation of haematopoietic stem cells. Ann Transplant 3: 5-13, 1998.

30. Hanyu A, Kojima K, Hatake K, et al: Functional in vivo optical imaging of tumor angiogenesis, growth, and metastasis prevented by administration of anti-human VEGF antibody in xenograft model of human fibrosarcoma HT1080 cells. Cancer Sci 100: 2085-2092, 2009.

31. Yu S, Qin D, Shangary S, et al: Potent and orally active smallmolecule inhibitors of the MDM2-p53 interaction. J Med Chem 52: 7970-7973, 2009.

32. Liang WC, Wu X, Peale FV, et al: Cross-species vascular endothelial growth factor (VEGF)-blocking antibodies completely inhibit the growth of human tumor xenografts and measure the contribution of stromal VEGF. J Biol Chem 281: 951-961, 2006.

33. Braczkowski R, Schally AV, Plonowski A, et al: Inhibition of proliferation in human MNNG/HOS osteosarcoma and SK-ES-1 Ewing sarcoma cell lines in vitro and in vivo by antagonists of growth hormone-releasing hormone: effects on insulin-like growth factor II. Cancer 95: 1735-1745, 2002.

34. Vihinen P, Riikonen T, Laine A, et al: Integrin alpha 2 beta 1 in tumorigenic human osteosarcoma cell lines regulates cell adhesion, migration, and invasion by interaction with type I collagen. Cell Growth Differ 7: 439-447, 1996.

35. Tainsky MA, Shamanski FL, Blair D, et al: Human recipient cell for oncogene transfection studies. Mol Cell Biol 7: 1280-1284, 1987.

36. Frese KK and Tuveson DA: Maximizing mouse cancer models. Nat Rev Cancer 7: 645-658, 2007.

37. Heremans H, Billiau A, Cassiman JJ, et al: In vitro cultivation of human tumor tissues. II. Morphological and virological characterization of three cell lines. Oncology 35: 246-252, 1978.

38. McAllister RM, Gardner MB, Greene AE, et al: Cultivation in vitro of cells derived from a human osteosarcoma. Cancer 27: 397-402, 1971. 